\section{MRS Spring Meeting Stretches Across Disciplines}

Technical content of the 1997 MRS Spring Meeting, with 26 technical symposia, stretched from polymers to superconductors, polycrystalline thin films to epitaxial growth mechanisms, and from applications in giant electronics and microelectronics to sports and recreation. The meeting, located at the San Francisco Marriott and chaired by David J. Eaglesham (Bell Laboratories, Lucent Technologies), Linda Griffith (Massachusetts Institute of Technology), and Alexander $\mathrm{H}$. King (State University of New York, Stony Brook), ran from March 31 to April 4. The meeting attracted over 2,500 registrants and gave an attendee-topaper ratio of 1.36 , a change from a trend toward a one-to-one attendee-to-paper ratio that had been developing this decade. This meeting also showed a high selectivity of presentations with a record $19 \%$ of submitted abstracts rejected.

The Meeting unveiled the first competition for the outstanding poster presentation at each of the meeting's three evening poster sessions. Sponsored by the Meeting Chairs, the nightly presentation at the winning authors' board included a congratulatory champagne toast with the Chairs, prominent display of their posters in the foyer throughout the week, and a cash award. (See sidebar on page 60.)

Paul S. Peercy, President, SEMI/ SEMATECH, gave the plenary presentation on Monday evening on "Materials Research and the Semiconductor Industry in the Twenty-First Century." Technology is moving rapidly through the information age and is heading into the biotechnology age, Peercy said. He set in this context the role of materials engineering and processing technology as an enabler of the convergence of communication, computing, and consumer electronics. He remarked that Moore's law-the phenomenon of the number of transistors per chip continuing to double every 18 months-is an economic statement, not a physics statement, driven by the industry's decision to put enough money into R\&D and other needs to maintain that rate of growth. Whether economic drivers or scientific shortcoming will knock the trend off that curve is not yet clear. Roadmaps to stay on that curve suggest smaller feature sizes, larger wafers, and innovative ideas such as moving to copper metallization and new low-dielectric-constant materials as other usual methods of

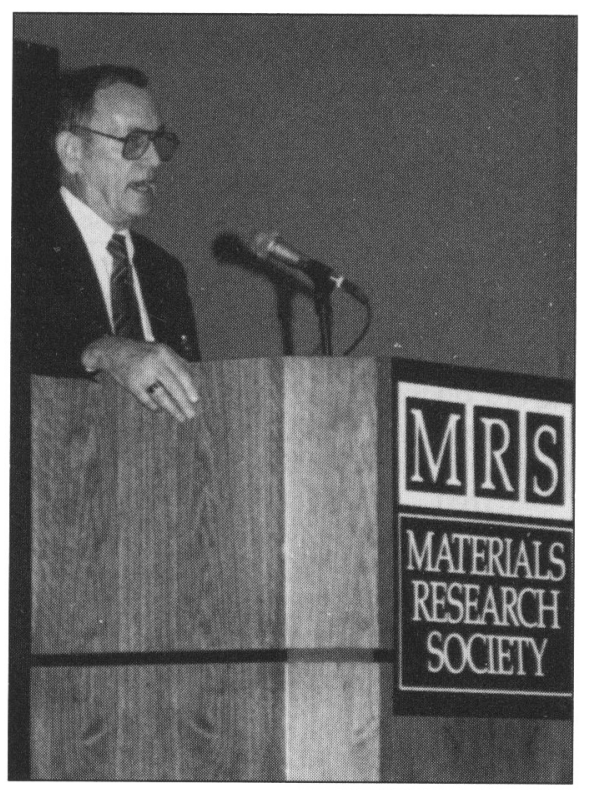

Paul S. Peercy, President of SEMISEMATECH, gave the plenary presentation on "Materials Research and the Semiconductor Industry in the Twenty-First Century" at the 1997 MRS Spring Meeting in San Francisco.

improvements, such as yield improvements, reach their limits. Peercy also addressed how the method of getting research done is likely to change. Instead of R\&D and all of the development work done in one institution, the research environment has become stratified, with different institutions or sectors involved in complementary parts of the technology development cycle as companies opt to outsource work instead of doing it all inhouse. Substantial and delicate interaction among the various institutions is essential to optimize use of knowledge and resources and to generate effective results. An article based on Peercy's presentation is scheduled to appear in a future issue of MRS Bulletin.

Prior to the plenary presentation, graduate student awards were given to four of nine graduate student award finalists.

The 1997 MRS Outstanding Young Investigator Christopher N. Bowman of the University of Colorado gave a presen(See photo on page 56.) tation on "Polymerization and Properties of Polymer-Stabilized Ferroelectric Liquid Crystals." To overcome the limited switching speeds of twisted nematics liquid crystals, ferroelectric liquid crystals have been considered, but they are susceptible to mechanical shock. Bowman's presentation addressed how polymers can be used to stabilize the ferroelectric liquid crystals, and how various factors affect the electrooptic behavior. The polymers can be introduced into the liquid crystals as monomers which are then photopolymerized. Bowman found that the temperature and ferroelectric-liquid-crystal phase of polymerization affects both polymerization behavior and electro-optic properties. The normalized polymerization rate increases for monomers as the order of the liquid crystal phase increases. Also, segregation is dramatically different depending on chemical structure. Bowman explained that if diffusional limitations of the growing polymer chains in the liquid crystal are significant, then the polymerization rate increase is driven by termination rate suppression. If diffusional limitations are not significant, the rate increase is driven by segregation phenomena. The material behavior can be modeled by knowing the segregation characteristics. An article based on Bowman's presentation is scheduled to appear in a future issue of MRS Bulletin.

Presented at noontime, Symposium $X$, Frontiers of Materials Research: Authoritative Reviews for Nonspecialists, included a clever array of topics geared toward a broad technical audience. Monday covered life on Mars with one talk on a Martian meteorite and another on robots for Mars. Tuesday started with "What's New in the Sausage Factory," focusing on how the government works, with a second talk on university and industry research. Wednesday covered novel materials with negative aspect ratios (thermal expansion and Poisson's ratio). The Thursday session addressed "Materials Challenges in the Human Genome Project." (See sidebar for a summary of these presentations.)

A Public Affairs Forum was held to solicit input on an NRC study on materials physics, a continuation in a series of meetings previously held at the 1996 MRS Fall Meeting and the 1997 March Meeting of APS. For more information on this study, see the March 1997 (page 60) and June 1997 


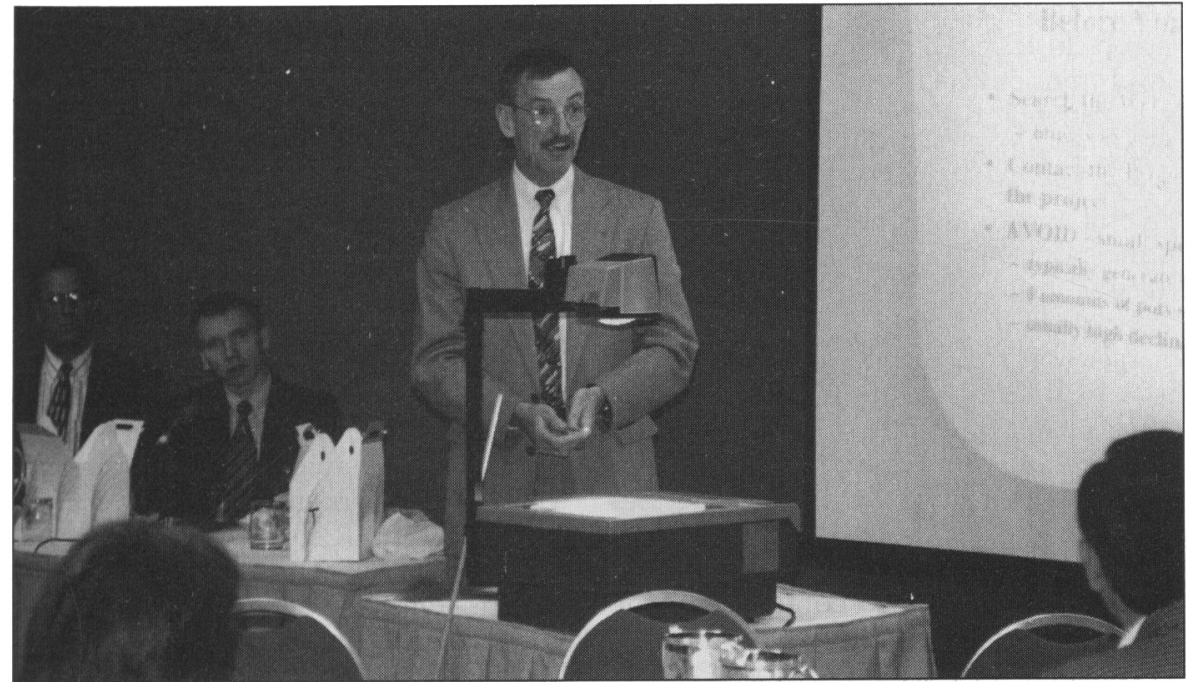

Thomas Weber (NSF) advises attendees interested in seeking research funds from NSF He was one of three panel members discussing grantsmanship at the Junior Faculty Forum held at the 1997 MRS Spring Meeting. Seated from right to left are John Pazik (ONR) who compared and contrasted the review process and funding opportunities at ONR and NSF and Mark Goorsky (UCLA) who discussed the benefits and complications of industrial funding for academic research.

(page 9) issues of MRS Bulletin and the Condensed Matter and Materials Physics website at http: / / www2.nas.edu/ $\mathrm{cmmp} /$.

The MRS Membership and Academic Affairs Committees sponsored a Junior Faculty Forum on the art of obtaining grants, a student mixer, a networking happy hour for job seekers, and a job center. University chapters and student teams were invited to participate in a photo contest of "symmetry scapes," with photos displayed during the meeting. A Women in Materials Science and Education (WIMSE) event focused on couples managing a dual-career family. About 50 people participated in a lively discussion on their individual experiences. Attendees represented all areas of employment including industry, academia, and government laboratories. Experiences ranged from universities hiring both members of a couple, spouses finding jobs in the same company to companies who did not allow employees to be married to each other. Some best practices were shared and time was allowed for attendees to informally network with each other. WIMSE, an informal group within MRS, is dedicated to promoting women in the field of materials science and engineering. The group typically meets at 7:00 a.m. on Tuesday at each conference, and welcomes everyone to participate.

A full exhibit attracted many visitors, while tutorials given by leading experts lured over 500 participants.

In Symposium A, techniques were introduced to help understand the growth and physical properties of hydrogenated amorphous silicon (a-Si:H), particularly the role of dangling bonds. Amorphous siliconbased solar cells continue to increase their efficiency, with the announcement of a stabilized conversion efficiency of $13.0 \%$ in a triple junction of amorphous $\mathrm{Si} / \mathrm{Si}-\mathrm{Ge} / \mathrm{Si}-$ Ge. A particularly novel application of amorphous silicon is a device for restoring

\section{ACRONYM KEY}

AFB: air force base

AMD: advanced micro devices

AMLCD: active-matrix liquid crystal

display

ANL: Argonne National Laboratory

APS: American Physical Society

ARO: Army Research Office

CMP: chemical mechanical polish

CMR: colossal magnetoresistive

CMU: Carnegie Mellon University

$C$-V: capacitance-voltage

DARPA: Defense Advanced Research

Projects Agency

ECR: electron cyclotron resonance

ESR: electron spin resonance

FET: field-effect transistor

FIB: focused ion beam

GE: General Electric Corporation

GM R\&D: General Motors Research and

Development

HBT: heterojunction bipolar transistor

HP: Hewlett-Packard Laboratories

HTS: high-temperature superconductor

ICP: inductively coupled plasma

IR: infrared

LANL: Los Alamos National Laboratory
PECVD: plasma-enhanced chemical vapor deposition

p-HEMT: pseudomorphic high electron mobility transistor

PIPS: polymerization induced phase separation

PNNL: Pacific Northwest National Laboratory

R\&D: research and development

rf: radio frequency

RIE: reactive ion etching

RTCVD: rapid thermal chemical vapor deposition

RTP: rapid thermal processing

SNL: Sandia National Laboratory

STM: scanning tunneling microscopy (microscope, micrograph)

TEM: transmission electron micrograph

TFT: thin-film transistor

TI: Texas Instruments

UC: University of California

UCLA: University of CaliforniaLos Angeles

ULSI: ultralarge-scale integration

UV: ultraviolet

VLSI: very large scale integration XPS: x-ray photoelectron spectroscopy 
vision by implanting behind the retina a photodetector array made on titanium foil. A joint session with Symposium $G$ on flatpanel-display materials covered low-temperature TFT processing by sputter deposition or PECVD which might enable lightweight, flexible active-matrix displays on plastic substrates. Symposium G also covered rapid improvement in PDLC and $\mathrm{H}$ PDLC liquid crystal materials which may enable full-color reflective displays with low power consumption and high contrast. In the area of field-emission displays, a 4-in. full-color field-emission display with 80 million tips was discussed.

The optical pump stimulated emission of conjugated polymers and organic materials has become a "hot" research area of organic electronics because of the potential development of electrically pumped organic diode lasers. This topic and research of organic-polymeric LEDs - the mainstream of organic electronic devices-was covered in Symposium H. Another highlight of this symposium was the study of device degradation; some devices have shown operation lifetimes of more than three thousand hours. Also an efficient blue-green electrochemical cell with $4 \%$ external quantum efficiency was demonstrated.

Compound semiconductors, the subject of Symposium C, are available in wafers of 6 in. $(150 \mathrm{~mm})$ diameter, pointing the way to cost reductions for high volume parts. Four-level metal interconnect schemes are prevalent, enabling digital circuits within excess of two million transistors. ICP approaches, with greater controllability of the plasma excitation, were described as the "method of the near future" versus RIE or ECR-type etching systems.

GaN, InN, AlN and their alloys, discussed in Symposium D, are now finding applications in short-wavelength lasers and high-power electronics. Nanotubes found in AlGaN/InGaN/GaN structures were found to tie in with reports that Incomposition modulations in the quantum-well give rise to quantum dots that may enhance stimulated light emission in lasers. A theoretical model for enhancing $p$-type doping in $\mathrm{GaN}$ by using oxygen co-doping with Be or $\mathrm{Mg}$ was presented. Increasing the $p$-type conductivity is a critical issue to reducing laser thresholds in this material.

Symposium E provided a snapshot of phenomena such as ion implantation, diffusion, and gettering, which are scientifically challenging yet important issues for processing of silicon devices. New experimental and theoretical insights have been presented on cluster formation and dissolution in ion-implanted crystalline $\mathrm{Si}$, casting new light on phenomena such as damage recov- ery, transient enhanced diffusion, and boron clustering. Techniques such as deeplevel transient spectroscopy, STM, and xray scattering have been successfully used to monitor defect evolution in $\mathrm{Si}$, providing precious information on vacancy-interstitial recombination, defect agglomeration, and doping clustering which can be used to validate simulation tools.

Symposium F delved into RTP's most persistent problem: temperature measurement. Equipment vendors are starting to provide useful solutions to this problem. A panel discussion following this session centered on which processes and at what time RTP will move into manufacturing. As an example, $\mathrm{Si} / \mathrm{Ge}$ by RTCVD is starting to come out of the research laboratory and is moving into production of devices.

In Symposium J, Materials Reliability in Microelectronics, a sandwich structure four-point bend test was described to measure adhesion between interlayer dielectric and metal interfaces. Nanoindentation techniques showed the advantage of using thin $\mathrm{Cr}$ and $\mathrm{W}$ layers to promote adhesion of copper thin films. Reliability of copper interconnect metallization and the importance of thin diffusion barriers such as $\mathrm{Ta}$ and TiSiN films were also considered. MOS gate dielectric reliability and oxide reliability testing were other important issues on the table. In a joint session with Symposium $\mathrm{P}$, interactions between surface preparation and gate oxide reliability were addressed. High-energy implantation was shown to effectively getter metal impurities, maintaining gate oxide reliability despite intentional $\mathrm{Fe}$ contamination.

A special session in Symposium $P$, Science and Technology of Semiconductor Surface Preparation, was devoted to cleaning associated with chemical-mechanical polishing. The symposium also covered surface roughening, comparison of different solvents, and gate oxide integrity. The presentations indicated that in a production environment, many different cleaning sequences result in similar oxide integrity. The Symposium also had a session on environmentally friendly processing, including dilute $\mathrm{SC}-2$ processing and ways to reduce rinsing water.

Symposium L, Epitaxial Oxide Thin Films, covered materials ranging from epitaxial ferroelectric thin films and high-temperature superconductors to CMR oxide thin films. An oxide epitaxial lift-off technique was described for the integration of complex oxides with other materials, thus broadening application possibilities previously limited by epitaxial growth constraints. An exciting breakthrough on the road to UV solid state lasers was the demonstration of room temperature UV lasing in epitaxial zinc oxide films.

A session on nano- and microstructure and magnetic properties in Symposium M, Magnetic Uitrathin Films, Multilayers, and Surfaces, emphasized the relationship of the magnetic properties of films to their structure determined by probe and other microscopies. Significant progress has been made in the physical description of magnetic reversal in very small structures using new nanofabrication and physical observation methods such as magnetic force microscopy and the magneto-optical Kerr effect.

Symposium $\mathrm{R}$ covered Materials Issues Related to Development of Textured High-Temperature Superconducting Conductors. All of the methods of preparing highly textured, high-current-density $\mathrm{YBa}_{2} \mathrm{Cu}_{3} \mathrm{O}_{7}$ (Y123) deposits on flexible metal substrates have in common epitaxial deposition of the superconductor on a highly textured oxide buffer layer on a metal substrate to obtain a highly textured superconducting film which has few weakly linked high-angle grain boundaries. The various approaches are distinguished by the method used to obtain the textured buffer layer. Approaches which have led to high-current-density films include ion-beam-assisted deposition of the buffer, epitaxial deposition of the buffer on highly textured metal strip, and a method developed in Japan in which biaxially textured buffers are obtained by pulsed laser deposition by inclining the substrate relative to the ablation plume.

Organic and inorganic materials, liquid crystals, photorefractive materials, particle suspensions, and biological materials were all covered in Symposium S, Materials Optical Limiting. Progress showed in new material design by computation, nonlinear optical propagation codes for designing devices, and demonstration devices validated in actual sensor protection tests. IR optical limiting technology is approaching a stage of maturity with results reported for thermochromic and semiconductor materials.

The thrust of Symposium T, Computational Materials Science at the Mesoscale, was on the development of new methods to describe materials behavior at the microstructural level. An important focus was on linking computational time and length scales. Nucleation, dendritic solidification, and coupling macroscopic response to microscopic properties were three key topics reported.

Symposium W, Metastability and Critical Phenomenon in Polymer Phase Behavior, showed how polymers, because of their relatively slow kinetics and large 
length scales, lend themselves to fundamental studies of phase transitions and design of technologically important structures. Crystalline polymers showed sizedependent metastability, thus a phase which is metastable for an infinite size can become the stable phase when sufficiently small. Other work showed how photo polymerization using crossed laser beams can set up a spatially varying curing condition in an initially homogeneous nematic blend of monomer and liquid crystal.

A major focus of Symposium $Z$, The Workshop on Specimen Preparation for Transmission Electron Microscopy of Materials, was ion-beam techniques, and their applications to semiconductors, steel, and multilayer samples. An open forum held at the end of the symposium migrated toward the topics of sample contamination and FIB.

Other topics covered included Epitaxial Growth-Principles and Applications, Polycrystalline Thin Films, Multilevel Metal Process Integration, Low-DielectricConstant Materials and Applications in Microelectronics, Materials / Failure Analysis for Silicon ULSI Processing, Thermoelectric Materials-New Directions and Approaches, Rapid Prototyping and Solid Freeform Manufacture, Interfacial Effects and Organization of Inorganic-Organic Composite Solids, and Materials in Sports and Recreation.

See the following pages for more complete summaries of the symposia. Also see the MRS website http: / / www.mrs.org/ for more detailed program information and abstracts of presentations.

\section{Research on a-Si, mc-Si Spans from Fundamentals to Subretinal Implants (See MRS Proceedings Volume 467)}

Hydrogenated amorphous silicon (a$\mathrm{Si}: \mathrm{H})$ continues to pose fascinating materials problems at the same time as it is finding ever new applications. Symposium A, Amorphous and Microcrystalline Silicon Technology, reflected this range from basic research to new uses. The symposium also encompassed microcrystalline silicon (mc-Si), which can be grown by making comparatively small changes to the deposition conditions for amorphous silicon. Microcrystalline silicon often is a two-phase mixture of amorphous and crystalline material. E. Bertran et al. (Barcelona University, Spain and CNRS, France) clearly documented this structure with TEMs of dispersions of silicon nanocrystals in matrices of a-Si:H. Current understanding of $\mathrm{mc}-\mathrm{Si}$ is where it stood with a-Si: $\mathrm{H} 15$ years ago.

Techniques were introduced to help understand the growth and physical

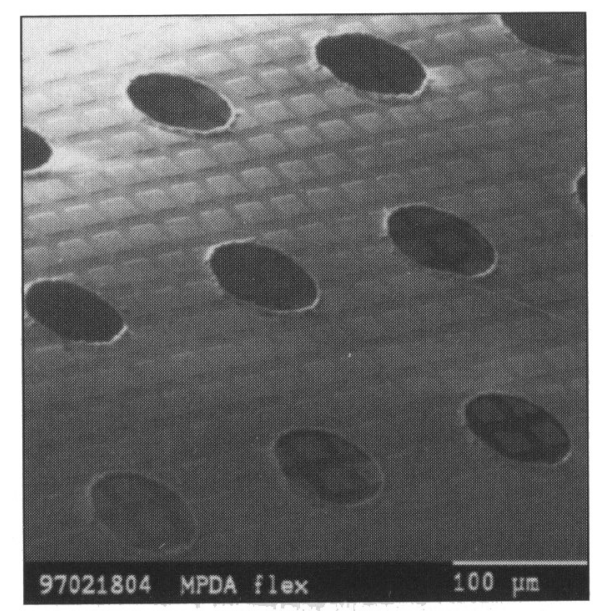

M. Schubert (Univ. of Stuttgart), in Symposium A on Amorphous and Microcrystalline Silicon Technology, described a device for restoring vision lost by the decay of photoreceptor cones. An a-Si:H based photodetector array made on titanium foil is implanted behind the retina. Laser holes provide access for retina cell nourishment (the photoresist carrier can be seen in the holes prior to removal). This medical application takes advantage of the thinness and flexibility offered by a micrometer-thick a-Si:H device fabricated on a micrometer substrate.

properties of a-Si:H. S. Yamasaki et al. (JRCAT-NAIR, Japan) succeeded in taking ESR spectra of a-Si:H during deposition and identified two components of the dangling-bond signal, one building up and decaying quickly as the glow discharge is turned on and off, and the other decaying slowly. Using a micromechanical resonator, $R$. Crandall et al. (NREL and Cornell Univ.) reported that $\mathrm{a}-\mathrm{Si}: \mathrm{H}$ deposited by the hot-wire technique is the first material to depart significantly from the universal low-temperature internal friction of noncrystalline solids. Its internal friction can be lower by orders of magnitude. The Staebler-Wronski effect, which is the reversible, light-induced degradation of a-Si: $\mathrm{H}$, is gradually being revealed as an effect involving both the well-known increase in the density of silicon-dangling bonds, as well as broader structural changes. H. Fritzsche (Tucson, AZ) emphasized this point in his review talk. N. Hata (Electrotechnical Laboratory, Japan) presented data revealing a surprisingly large effect of light soaking on interband electroabsorption. These data suggest that light soaking first changes the network as a whole before bonds begin to break. M. Stutzmann
(Technical Univ. of Munich) described a technique for probing the interrelation of hydrogen and defects in amorphous silicon. The method uses electrically detected electron spin resonance at very low resonance frequencies (about $500 \mathrm{MHz}$ ); the use of such low frequencies leaves a line shape which is dominated by the hydrogen interactions. However, even with the new approach no light-induced effect on the line shape was found.

In research on an amorphous-siliconbased solar cell, J. Yang (United Solar Systems Corp.) announced a stabilized conversion efficiency of $13.0 \%$ in a triple junction of amorphous $\mathrm{Si} / \mathrm{Si}-\mathrm{Ge} / \mathrm{Si}-\mathrm{Ge}$. S.M. Gates (IBM) discussed the prospects for using plastic substrates for a-Si:H TFT circuits. He also announced the result of a collaboration with J. Abelson et al. (Univ. of Illinois-Urbana) on the fabrication of a-Si:H TFTs at a temperature of $125^{\circ} \mathrm{C}$, which helps pave the way to an era of electronics-on-plastic. Two groups (V. Chu et al., INESC and IST, Portugal, and R. Schropp et al., Univ. of Utrecht, Netherlands) reported the fabrication of TFTs from a-Si:H deposited by the hotwire technique, thus introducing to thinfilm electronics amorphous silicon having an unconventional network. M. Schubert (Univ. of Stuttgart) described a device for restoring vision lost by the decay of photoreceptor cones.

Symposium Support: NAPS France, Fuji, Solarex, Sanyo, dpiX, Voltaix, MV Systems, United Solar Systems, Sharp, EPRI, and Canon.

\section{Compound Semiconductors Move to Larger Scale Integration}

Symposium C, Processing of Compound Semiconductors for High-Speed Devices, showed that large diameter GaAs substrates (6 in. or $150 \mathrm{~mm}$ ) are now available from several sources, pointing the way to cost reductions for high-volume parts, and significant die per wafer increases for VLSI devices. Ion implantation processing continues as the mainstay for MESFET devices. MBE- and MOCVD-grown materials continue to contend in the higher frequency/high performance devices (pHEMTs, HBTs, and high-performance MESFETs). Fourlevel metal interconnect schemes prevail, enabling digital circuits in excess of twomillion transistor complexity.

High volumes of consistent, reproducible materials are a key issue as the industry shifts to production focus. Variations in $V_{\text {be }}$ as small as $3 \mathrm{mV}$ across an HBT wafer, and FET threshold control on the order of 20-50 mV wafer-to-wafer, were reported. Integrated optoelectronics using the power of selected area MBE 
growth were also an exciting topic

A substantial effort continues to be directed to etching and damage effects. A finding common to all of the etching investigations was the importance of ion energy in the plasmas, not the ion flux, in controlling the damage in the surface region. ICP approaches, with greater controllability of the plasma excitation, appear to be the "method of the near future" versus RIE or ECR-type etching systems. Surface and trap-related phenomena associated with $\mathrm{AlGaAs}$ in various device configurations may be circumvented with InGaP, InAlAs, and $\mathrm{InGaAs}$ materials.

Reliability of HBT devices continues to be a topic of debate: at low stress levels $\left(<40 \mathrm{kA} / \mathrm{cm}^{2}\right)$ Be-doped-base HBT devices were relatively long-lived and stable. Under high stress conditions (60-100 $\mathrm{kA} / \mathrm{cm}^{2}$ ), even the carbon-doped base devices fail rapidly due to migration of the acceptor species.

High-frequency microwave devices for frequencies above $\sim 40 \mathrm{GHz}$ are still the domain of InP materials. Devices with gate lengths below $0.1 \mu \mathrm{m}$ showed $f_{\mathrm{t}}$ values up to $300 \mathrm{GHz}$.

\section{Nitrides Advance and Diversify}

\section{(See MRS Proceedings Volume 468)}

Symposium D on Gallium Nitride and Related Materials focused on recent developments in $\mathrm{GaN}, \operatorname{InN}, \mathrm{AlN}$, and their alloys that are now finding application in short wavelength lasers $(\sim 400 \mathrm{~nm}, \mathrm{cw}$ at room temperature) and high-power electronics $(2.8 \mathrm{~W} / \mathrm{mm}$ at $8 \mathrm{GHz})$. S.P. DenBaars (UC-Santa Barbara) reported on advances in $\mathrm{AlGaN} / \mathrm{GaN}$ transistors with frequency performance metrics of $50 \mathrm{GHz}$ for $f_{t}$ and 92 $\mathrm{GHz}$ for $f_{\max }$ for a $0.2 \mu \mathrm{m}$ gate length. F. Ponce (Xerox-PARC) presented evidence of nanotubes in AlGaN/InGaN/GaN structures extending through the quantumwell regions. This ties in with reports that In-composition modulations in the quantum-well give rise to quantum dots that may enhance stimulated light emission in lasers. The topic of In-composition inhomogeneities was also addressed by J. Rosner (HP) who showed convincing cathodoluminescence images from the edge of InGaN quantum-well structures demonstrating inhomogeneous emission.

While much of the symposium reported on advances in material preparation and understanding of detect issues, similar advances in material processing were reported. S.J. Pearton (Univ. of Florida) presented an overview of the state of processing and future challenges with a particular emphasis on the technologies that will be required for realizing high-power devices in the group-III-nitrides. R.J. Shul

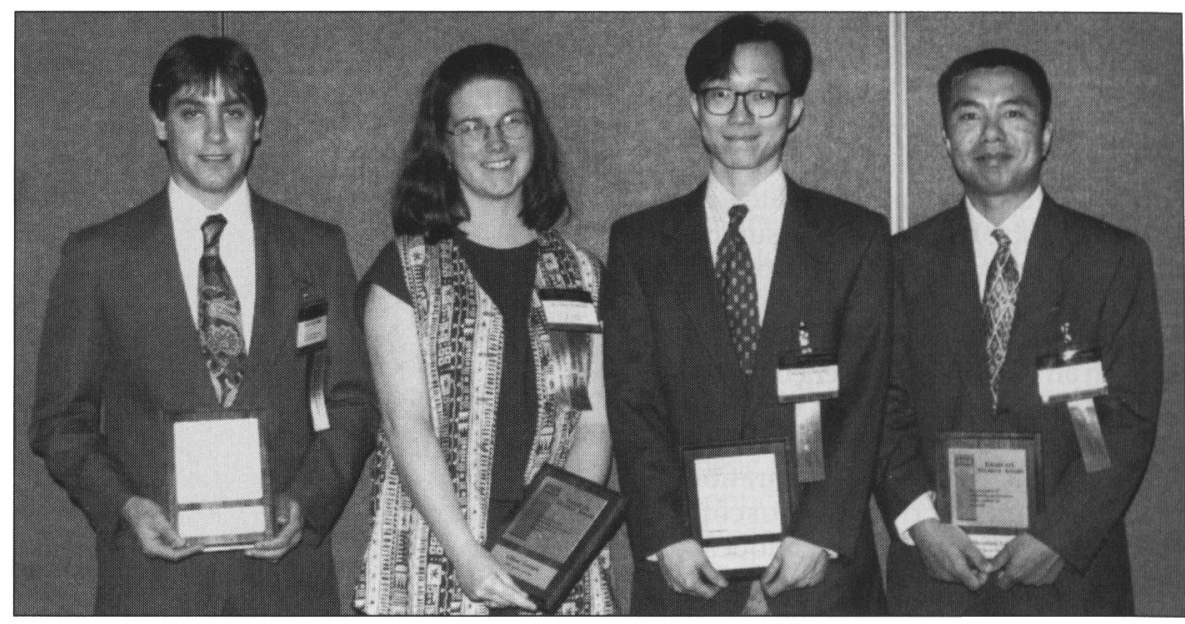

Four Graduate Student Awards were presented at the 1997 MRS Spring Meeting in San Francisco. (See "MRS Seeks Graduate Student Award Applications for 1997 Fall Meeting," this issue.) Graduate Student Award recipients are (left to right) Eric B. McDaniel (University of Virginia), Gillian R. Goward (University of Watertoo), Chung-chih Wu (Princeton University), and Shouliang Lai (University of Illinois-Urbana).

(SNL) presented recent results for dry etching these materials in high-density plasmas with etch rates and facet morphologies that should be applicable to laser formation. Q.Z. Liu (UC-San Diego) reported on thermally stable PtSi and NiSi Schottky contacts to GaN that appeared attractive for high-temperature electronics.

Some noted theoretical results were given by $W$. Chow (SNL) on the gain in laser structures. He suggested that reabsorption in nonhomogeneous InGaN quantum-well layers (e.g., quantum dots) may cause an increase in the laser threshold currents. Significant discussion was generated by T. Yamamoto (Osaka Univ.) with his theoretical model for enhancing $p$-type doping in GaN by using oxygen co-doping with Be or $\mathrm{Mg}$. Increasing the $p$-type conductivity is a critical issue to reducing laser thresholds in this material.

Symposium Support: ARO.

\section{Ion Implantation Presents Challenges in Si Processing}

(See MRS Proceedings Volume 469)

Symposium E on Defects and Diffusion in Silicon Processing was new to the MRS meetings. The choice of such a specific subject has resulted in a lively and wellattended symposium, providing a snapshot of phenomena such as ion implantation, diffusion, and gettering, which are scientifically challenging and of great importance to the processing of silicon devices. The development of reliable, predictive, and quantitative simulation tools, necessary for the design and fabrication of state-of-the-art microelectronic devices, requires a synergy between experiments and simulations for the proper assessment of defect and impurity behavior in silicon. Experimental and theoretical insights have been presented on cluster formation and dissolution in ion-implanted crystalline $\mathrm{Si}$, casting light on phenomena such as damage recovery, transient enhanced diffusion, and B clustering. Techniques such as deep-level transient spectroscopy, STM, and $x$-ray scattering have been successfully used to monitor defect evolution in $\mathrm{Si}$, providing information on vacancy-interstitial recombination, defect agglomeration, and doping clustering which can be used for the validation of the simulation tools. Simulation methods, using a combination of kinetic Monte Carlo with parameters derived from $a b$ initio calculations, have been shown to hold high promise for the development of quantitative and predictive simulation tools. Low-energy ion implantation will be able to provide the shallow junctions needed for the next ULSI generation of devices. At the same time, the use of low energy beams has revealed interesting phenomena such as the room temperature diffusion of selfinterstitials, vacancies, and B atoms. These room-temperature-diffusion phenomena need to be taken into account for the proper simulation of defects and diffusion in silicon processing.

Symposium Support: LLNL; Philips; SEMATECH; Technology Modeling Assoc.; Bell Labs, Lucent Tech.; SGS Thomson Microelectronics; and Italian National Council of Research. 


\section{Frontiers Symposium Reaches Outer Space, Genetic Engineering, Perversity, and Funding}

Researchers often produce materials designed to provide special properties needed by engineers. Less frequently, they stimulate new engineering by creating new materials with novel properties. Both of these types of materials development were addressed in the 1997 MRS Spring Meeting's Symposium X, along with the outlook for funding to keep all sorts of materials research going.

Each of the four sessions in Symposium $X$ was devoted to two lectures on closely related topics. Monday's talks addressed the extraterrestrial frontier of Mars, even is a new probe was en route to the red planet. Mars is a little smaller than Earth, but it has some large-scale structures: One of its canyons would stretch all the way across the United States, and much of the planet's geography is dominated by what seem to be dry river-beds in familiar-looking drainage patterns, suggesting the one-time existence of water, and thus the possibility of life. In some places, snow is on the surface. Even at Mars's distance, you can occasionally just wait for the specimens to come to you and the first talk, by Stanford University's S. Clemett, gave a detailed description of the analysis of the meteorite ALH84001. This is the small piece of Martian debris that fell to Earth and was collected in Antarctica, later providing a first direct indication of the possibility of life on Mars and sparking massive controversy in the scientific and popular press. All of the evidence obtained so far derives from the analysis of the materials on the meteorite, and, as with many materials analysis reports, they tantalize but do not quite tell us everything we need to know. Addressing a technically literate audience, Clemett was able to discuss the many analytical techniques that have been applied to the meteorite-most of them familiar to materials researchers-and provide a clear discussion of what is and is not conclusive. JPL's F. Grunthaner provided a complementary talk on the development of micromechanical robots for Mars exploration, explaining the goals of the project, the extreme conditions under which the devices must operate, and the high expectations for their operation. News of the Mars Explorer mission will probably be announced about the time that this report is published. As with all extreme engineering challenges, materials determine how well the tools perform. When researchers get to their labs in July and turn on a spectrometer, they should consider the operation of a similar tool on Mars-it has to survive blast-off, travel, and landing; it has to obtain its own specimens in an uncontrolled environment; collect spectra and transmit the data to its operator, without the intervention of any technician, all within a strict weight budget. The whole thing would fit in a desk drawer, and there is no maintenance agreement with the manufacturer.

Another session of the Symposium was devoted to the technology of gene analysis-the means by which it is possible to read out the sequence of chemical groups along the famous double helix of the DNA molecule. Nature can transcribe sequence of these groups onto RNA molecules which then become the templates for proteins, but our tools are less elegant. Only four distinct groups, designated $a, c, g$ and $t$, are used to encode the genetic information, and biologists identify the existence of particular sequences of these groups by creating equivalent sequences of their antibodies. When a sequence on a protein or a nucleic acid meets its antibody sequence, they attach together, making it possible to identify the existence of that particular sequence in a specimen. The problem is to test unknown genes against large arrays of known sequences quickly and reliably. Deval Lashkari of Synteni, Inc. and Mark Chee of Affymetrix, Inc. (both Palo Alto, CA, companies) explained how test arrays holding many different antibody sequences can be made using, in part, lithographic techniques familiar from semiconductor pro- cessing. Although the scales of these devices lag far behind the semiconductor industry, some variant of Moore's law seems to be in effect with ever denser test arrays, in familiar multiples of $2 n$, scheduled for production over the next few years. Specialized systems are envisaged, to test for genes associated with diseases such as Alzheimer's, and a whole "lab on a chip" is under development for the parallel testing of a number of different gene sequences right in the doctor's office. Both speakers, by the way, made open invitations for materials scientists to submit their resumes.

Possibly revealing a perverse nature of their own, Meeting Chairs David J. Eaglesham, Linda Griffith, and Alexander H. King devoted one session of Symposium $X$ to talks on materials with perverse properties. Oregon State University chemist Art Sleight demonstrated how it is possible to conceive of materials having negative thermal expansion coefficients by combining stiff interatomic bonds with relatively flexible bond angles. These requirements can sometimes be found in crystals that contain corner- or edge-bonded oxygen tetrahedra, for example. He reviewed a number of materials that provide these properties in two dimensions, giving anisotropic thermal expansion, culminating with a description of the recently discovered three-dimensionally negative-TCR material, zirconium tungstate. An animated simulation demonstrated the rotational thermal vibrations that generate the negative thermal expansion. Ken Evans of England's University of Exeter demonstrated how similar ideas can lead to materials with negative Poisson ratios. The figure (p. 58) gives an idea of how these work, and provides a design for a simple model that can be constructed from paper strips, to provide some distraction and possibly even insight. (Try bending or flexing your model to see some unusual behavior.) The acute nodes that generate this behavior can be made macroscopically in honeycomb structures, as illustrated, or on a smaller scale in foams and cellular materials formed under special conditions, and the search is now on for materials in which atomic bonds form the necessary nodes. Materials with this property (called "auxetic materials") may find many applications, only some of which were hinted at. Among other things, it is easy to push an auxetic material into a tightly-fitting hole, but hard to pull it out again, making it useful as a fastener. Evans noted that cork has a Poisson ratio close to zero, which helps to explain why it is so hard to pull a partly inserted cork out of a wine bottle, and yet it is so easy for the bottler to push the cork in. Try a rubber bung to see the difference!

The remaining session of Symposium $X$ was devoted to one of the real frontier issues: obtaining the means to do research. The APS's Washington watcher, Robert Park, described how the U.S. federal budget is developed, describing who gets input into the process, how, and when. In his own cynical yet upbeat way, he attempted to address his assigned topic of "how the government works," pointing out that the title embodied a debatable assumption. Parks clearly distinguished between the ways things are supposed to work and the ways they actually do, noting the ineffectiveness of some parts of the process and certain groups whose role is nominally to provide input and advice. He explained the difference between budgets and appropriations and, most importantly, showed how, when, and where the system is open to external input that can be very effective if provided properly. Individuals and society officers should take note. Sheldon Weinig, now retired from a Vice Presidency at the Sony Corp. of America, provided an uninhibited perspective on university-industry collaborations. Noting that governmental support for basic research is shrinking, and that industrial basic research is also rapidly waning, he sought to answer the question Continued next page 


\section{Frontiers Symposium Continued. . .}

of how to support the basic science that will underpin new generations of technology in all fields. Weinig made a number of pointed observations about the behaviors of both industry and academia, and ended with three rather challenging recommendations: (1) Both sides need an attitude adjustment, with industry concentrating more upon the intellectual means by which it earns money, and academia being more sensitive to the ends of its intellectual product. Universities need to become much better at cost control in their research endeavors. (2) Industry must view academia as part of its supply chain and act accordingly. Universities provide both knowledge and people to industry, and companies should work with the universities to improve the quality and reduce the cost of both products just as they do with their other suppliers. Radically, Weinig suggests that companies should pay the universities for every graduate that they hire since this would provide a direct incentive to provide appropriately trained individuals. (3) With the pre-obsolescence half-life of technological knowledge shrinking rapidly, universities must devote more of their resources to providing retraining programs to industry, notably through distance-learning technology.
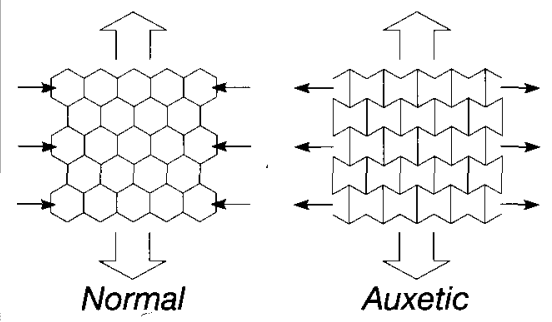

Honeycomb models of normal (positive Poisson ratio) and auxetic (negative Poisson ratio) materials. Those who remember what they learned in kindergarten will be able to construct these from craft paper, with a little patience. Pulling vertically on the normal model, as shown, makes it contract sideways. The same force on the auxetic material makes it expand. Bending the two models out of their plane produces strikingly different responses, which we leave to the reader to investigate.
RTP Matures with Stable and Production-Worthy Processes (See MRS Proceedings Volume 470)

Symposium F, Rapid Thermal and Integrated Processing, started with an excellent and well-attended tutorial given by F. Roozeboom (Philips Research Labs., Netherlands) and P.J. Timans (AG Assoc.). The tutorial included a good mixture of history, theory, and a review of practical applications for both novice and experienced RTP users.

The symposium itself began with a lively group of papers on RTP's most persistent problem: temperature measurement. Researchers were in general agreement that the equipment vendors are providing useful and production-worthy solutions to this problem, but D. DeWitt (NIST) in his invited paper warned that little attention is being given to aligning RTP temperature measurement with the International Temperature Scale and this will become a serious issue as metrology requirements continue to become tighter. The most important conclusion in the next session covering RTCVD is that $\mathrm{Si} / \mathrm{Ge}$ by RTCVD is starting to come out of the research area and be used in real production devices. Work in other interesting systems such as silicides, $\mathrm{SiC}$, and dielectrics is also progressing.

Mike Pas's (TI) invited papers gave views on which processes RTP is best suited for and when furnaces will continue to be the thermal method of choice. A couple of papers on fast ramp vertical furnaces provided insight into this rapidly growing competition to RTP. J.J. Wortman (North Carolina State Univ.) led the panel discussion following this session. The major topic again seemed to be for which processes and when RTP will be moved into manufacturing.

The integrated processing session showed that several groups are working on gate stack clusters with integrated precleaning and the results are encouraging. The silicide and annealing sessions pointed out that some important areas of study are still needed to fully understand these processes that have used RTP for many years. In the shallow junction area, studies on ambient control and interaction between the implant and anneal processes were very interesting. Several intriguing developments were discussed in the dielectrics sessions. M. Green (Bell Labs, Lucent Tech.) discussed the formation of nitride oxides in pure nitrogen instead of the customary $\mathrm{N}_{2} \mathrm{O}$ or $\mathrm{NO}$. Several papers looked into the problems of using the oxides of nitrogen to grow dielectrics with the proper nitrogen distribution. In the final session, several interesting applications were discussed as well as an excel- lent invited paper by $Y$. Nissim (CNET $\mathrm{Lab}$, France) which described production applications of RTP for surface passivation in the manufacture of III-V devices.

Symposium Support: Applied Materials, CVC Products, Vortek Industries, SensArray, ASM Europe, ASM America, Eaton Thermal Processing Systems, CGS Thermodynamics, AST Elektronit, and Dainippon Screen Manufacturing.

\section{New Emitters and Phosphor Reliability Advance Area of Field Emission Displays \\ (See MRS Proceedings Volume 471)}

Symposium G, Flat Panel Display Materials and Large-Area Processes, began with a tutorial given by T-J. King (UC-Berkeley) and B. Gnade (DARPA). Excellent overviews of the various display technologies were given which included in-depth discussion of TFT processes for AMLCD's and field emission displays.

Three days of symposium sessions were highlighted by a combined session with Symposium A. One theme of this session was the subject of low temperature TFT processing by sputter deposition or PECVD which might enable lightweight, flexible active-matrix displays on plastic substrates (S.M. Gates of IBM and C. Weber of Intel).

In other sessions, G.P. Crawford (Brown Univ.) discussed the rapid improvement in PDLC and H-PDLC liquid crystal materials which may enable full color reflective displays with low power consumption and high contrast. The area of field-emission displays is moving forward with work on new emitters (J. Robertson and W.I. Milne of Cambridge Univ.) and phosphor reliability (C.H. Seager, D.R. Tallant, and W.L. Warren of SNL). A four-inch full color field-emission display with 80 million tips was discussed by J. Kim (Samsung). In the area of phosphors, a combinatorial synthesis technique was presented (X-D. Sun of LBNL and X.D. Wu of Symyx) in which a large library of phosphor samples is generated and characterized in situ. This allows fast optimization of phosphor characteristics such as chromaticity and luminance. Also, interesting work was described on the application of nanocrystalline phosphors to display technology (R. Bhargava of Nanocrystals Tech.).

Symposium Support: Materials Research Group, Dow Chemical, Applied Komatsu Tech., Sharp Microelectronics Tech., Eastman Kodak Co., Xerox $P A R C$, and Corning.

\section{Organic Electronics Moves Closer to Applications}

Symposium H, Organic Electronic Materials and Devices, was solely devoted to the rapid growth in this area. Among sev- 
eral developments reported, the optical pump stimulated emission of conjugated polymers and organic materials has become one of the hottest research areas of organic electronics because of the potential development of electrical pumped organic diode lasers. Groups from UC-Santa Barbara (A.J. Heeger), Univ. of Sheffield (D.D.C. Bradley), Univ. of Arizona (N. Peyghambanan), and Bell Labs, Lucent Tech. (A. Dodabalapur) presented their results on stimulated emission from polymers and organics. The research of organic-polymeric LEDs remains the mainstream of organic electronic devices. Device-degradation mechanism study, which is critical to future practical application, is another highlight of this symposium. Y. Sato (Mitsubishi Chemical) summarized the latest development of device degradation studies; his device reaches more than three thousand hours of operation lifetime. In the device performance, $Q$. Pei (UNIAX) demonstrated an efficient blue-green electrochemical cell with $4 \%$ of external quantum efficiency and higher than 10 lumen/watt luminous efficiency. Organic transistor is another interesting area which attracted considerable attention. Z. Bao (Bell Labs, Lucent Tech.) demonstrated a solution processable regioregular poly (3-alkylthiophene) with high carrier mobility. Novel organic electronic devices such as light amplification devices, photovoltaic cells, and optical sensor arrays were also reported.

Symposium Support: NSF, Sanyo Vacuum Industries, Universal Display, Chemipro Kasei Kaisha, Futaba, IMES Co., UNIAX, Sumitomo Electric Ind., TI, dpiX, HP, and Xerox-PARC.

\section{New Materials and Problems Appear for Microelectronics Reliability (Seo MRS Proceedings Volume 473)}

Prior to the technical sessions of Symposium J on Materials Reliability in Microelectronics, P. Besser (AMD), J. Sanchez (Univ. of Michigan), and $\mathrm{O}$. Kraft (Stanford Univ.) presented a well-attended tutorial on the role of mechanical properties and microstructure in the reliability of advanced microelectronic interconnect systems. The Symposium covered a broad spectrum of topics on interconnect and gate dielectric reliability. The symposium started with a session on adhesion and fracture in interconnect systems, which included discussion of new measurement techniques, as well as new test results. Q. $\mathrm{Ma}$ and co-workers (Intel and Stanford Univ.) described development of a sandwich structure four-point bend test which was used to measure adhesion between interlayer dielectric and metal interfaces. Using nanoindentation techniques, $M$.

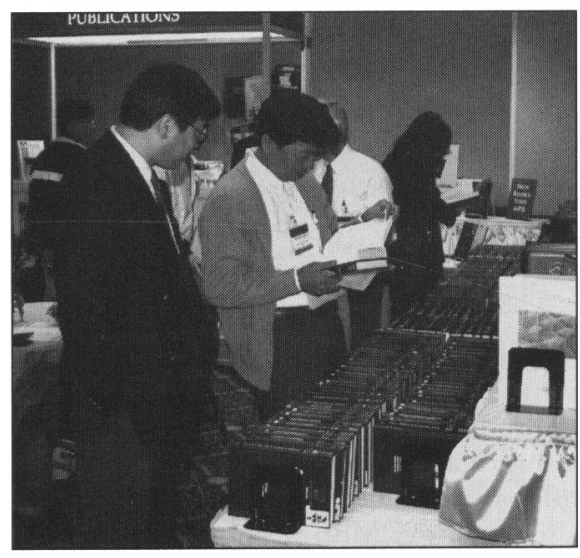

At the 1997 MRS Spring Meeting in San Francisco, MRS Publications offered an exciting new "bookstore" atmosphere for attendees to review and purchase proceeding volumes.

Kriese (Minnesota Univ.) reported on the efficacy of using thin $\mathrm{Cr}$ and $\mathrm{W}$ layers to promote adhesion of copper thin films.

With an eye toward the future, the reliability of copper interconnect metallization was the subject of several papers. S. Shingubara (Hiroshima Univ.) and S.H. Lee (Stanford Univ.) examined the dynamics of void formation and movement due to electromigration in copper lines. The importance of thin diffusion barriers in copper interconnect technology was emphasized in several presentations: $L$. Clevenger (IBM) reported on the effectiveness of thin Ta films, W.F. McArthur (UC-San Diego) discussed compositional effects on amorphous TiSiN, and D.J. Kim (Hanyang Univ.) investigated the effect of nitrogen concentration on amorphous TaSiN. J.A. Davis (Georgia Tech.) and J.D. Meindl (Georgia Tech.) introduced a methodology for wire length distributions in gigascale microprocessors, and showed how this might be used to define optimal multilevel interconnect architecture. Strong interest was shown in developments related to MOS gate dielectric reliability. Oxide reliability testing issues were raised in several talks.

T-C. Yang (Stanford Univ.) reported a dependence of oxide reliability on the anode Fermi-level for gate injection, and K. Catmull and co-workers (Motorola and Keithley) presented a comparison between $C-V$ measurements and noncontact test techniques.

Other noteable results included a discussion by H-C. Lin (Stanford Univ.) on the impact of the spatial wavelength of surface roughness on leakage current and reliability, and extensive evidence presented by D.L. Chapek (Micron Tech.) that the gate oxide reliability depends critically on the entire front-end process, including substrate material, wafer shape, field oxidation, polysilicon gate grain structure and in situ cluster-tool processing.

In a joint session with Symposium $P$ on Semiconductor Surface Preparation, interactions between surface preparation and gate oxide reliability were addressed. In a paper by S. Saito and co-workers (NEC and Bell Labs, Lucent Tech.), high-energy implantation was shown to effectively getter metal impurities, maintaining gate oxide reliability despite intentional Fe contamination.

T. Ito (Fujitsu) addressed the topic of UV-excited radicals for surface treatment, highlighting the smoothing effect that UV ozone had on preroughened surfaces. P. Mertens (IMEC, Belgium) demonstrated the importance of using C12-based chemistries during gate oxide growth, instead of $\mathrm{HCl}$, to maximize reliability while minimizing environmental impact.

Another joint session held with Symposium I on Polycrystalline Thin Films (see MRS Proceedings volume 472) focused on issues of common interest concerning microstructure, texture, and reliability. W.L. Brown (Bell Labs, Lucent Tech.) and C.V. Thompson (MIT) separately reviewed the effects of texture and grain-size distributions, respectively, on aluminum interconnect reliability.

In a session which featured novel measurement techniques, E.B. Varner (Intel) discussed methods for accurately measuring the thermal conductivity of thin-film interlevel dielectrics, which is important in determining joule heating effects in interconnects. Other sessions emphasized the effects of stress and stress relaxation in interconnects and the latest progress in the modeling of electromigration failure mechanisms.

Symposium Support: SNL, Aetrium /Sienna Tech., TOSHO SMD, Materials Research Corp., Lucent Tech. Inc., Tencor Instruments, Lucent Tech. Lab., Orlando, Mattson Tech., Digital Equipment, IBM T.J. Watson Research Center, and Motorola.

\section{Epitaxial Lift-Off Technique and Room- Temperature Lasing in UV Open Doors in Area of Epitaxial Oxide Thin Films (See MRS Proceedings Volume 474)}

In Symposium L on Epitaxial Oxide Thin Films, M. Eddy (Superconductor Technologies and UC-Santa Barbara) discussed an oxide epitaxial lift-off technique for the integration of complex oxides with other materials, making possible a wide variety of applications so far impossible due to epitaxial growth con- 
straints. M. Kawasaki (Tokyo Institute of Technology) demonstrated room-temperature lasing in UV in epitaxial zinc oxide films, an exciting breakthrough on the road to UV solid state lasers.

This Symposium focused on (1) epitaxi- al ferroelectric thin films, with M. Sinclair (SNL) reporting on optical characterization of lead-zirconate-titanate thin films and $J$. Roeder (Advanced Tech. Materials) discussing MOCVD of complex oxides by liquid delivery; (2) high-temperature super- conductor thin films, with B. Rappoli (NRL) presenting MOCVD precursor delivery data; J. Siejka (CNRS, France) discussing the mechanism of oxygenation of YBCO thin films grown via cathodic sputtering; J. Hsu (Univ. of Virginia) reporting

\section{Poster Prizes Awarded at the 1997 MRS Spring Meeting}

Lively, high-quality poster sessions are a highlight of a good meeting, providing opportunities for intense study, interaction, and feedback. Nobody sits through a poster presentation waiting for the turn of the paper they really wanted to see, so it is important for poster presenters to be able to catch and hold their audience with interesting science and an attractive presentation. With this in mind, 1997 MRS Spring Meeting chairs David J. Eaglesham, Linda Griffith, and Alexander H. King created a special first-ever "Meeting Chairs Poster Prize" to encourage high-quality presentations that benefit the presenter and audience alike. A single winner was announced at the end of each evening, and each winning poster earned a prize of $\$ 500$, a certificate, and the honor of being displayed in the main hallway for the remainder of the Meeting.

Although the MRS council demurred upon a name for the prize originally proposed by the Meeting Chairs, the criteria for the award ("content and presentation, weighted arbitrarily") were approved and rigidly observed. The Meeting Chairs judged the contest themselves, with advice and input from the organizers of the symposia represented at each poster session. That judging proved more difficult than anticipated, with many posters creating very strong impressions. Ultimately, however, winners had to be named from among the many that combined real excellence of both content and presentation. The winners would all have done well in a contest based solely upon either criterion, and were congratulated for the excellence of every aspect of their work. The Catholic University of Leuven deserves a special commendation for producing two of the three winners, amidst very strong competition.

All of the poster session participants were winners of a sort at the Spring Meeting, since the Meeting Chairs achieved another "first" by having the noted San Francisco local brew, Anchor Steam Beer, served at the open bars which, along with the buffet, are a popular feature of MRS poster sessions. Poster prize winners, however, deservedly drank champagne as seen in the accompanying photographs.

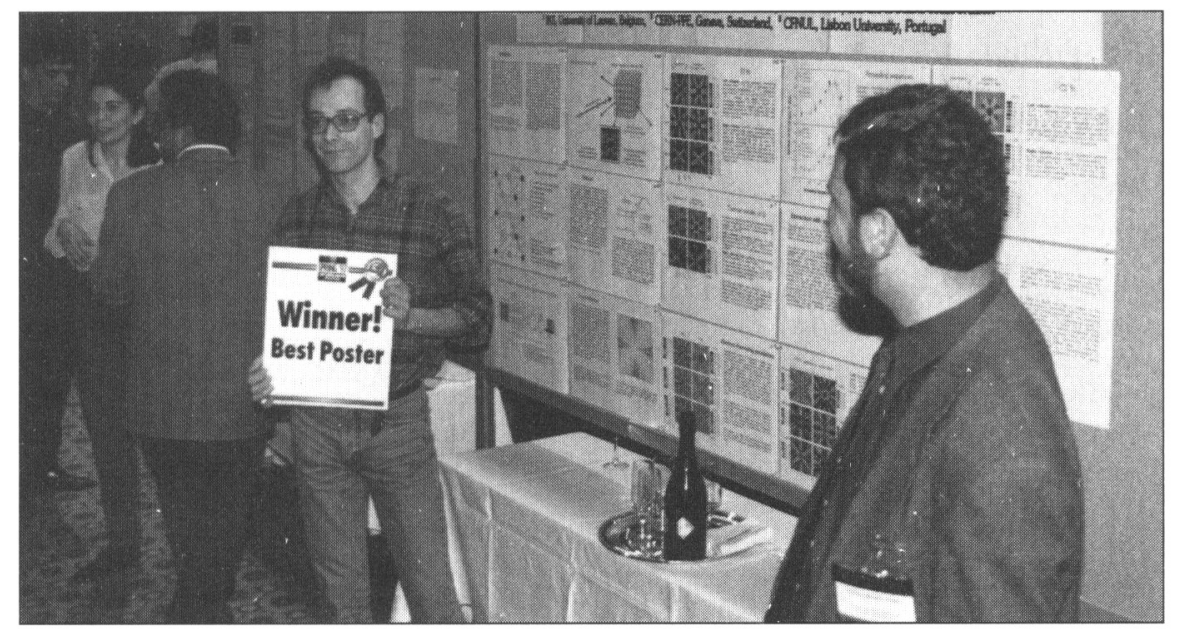

Wednesday (April 2) Poster Award recipient: Lattice Sites and Damage Annealing of Implanted Tm and Er In Si, Ulich Wahl, Katholieke Univ. Leuven, Dept of IKS, Leuven, Belgium; Joao Guilherme Correia, Lisbon Univ., Dept. of CFNUL, Lisboa, Portugal; Jo De Wachter, Guido Langouche, Katholieke Univ. Leuven, Dept of IKS, Leuven, Belgium; Jose Goncalves Marques, Lisbon Univ., Dept. of CFNUL, Lisboa, Portugal; Raf Moons, Andre Vantomme, Katholieke Univ. Leuven, Dept. of IKS, Leuven, Belgium; Isolde Collaboration, CERN, Dept. of PPE-IS, Geneve, Switzerland. (E7.8) (MRS chair Alexander H. King is seen to the right.)

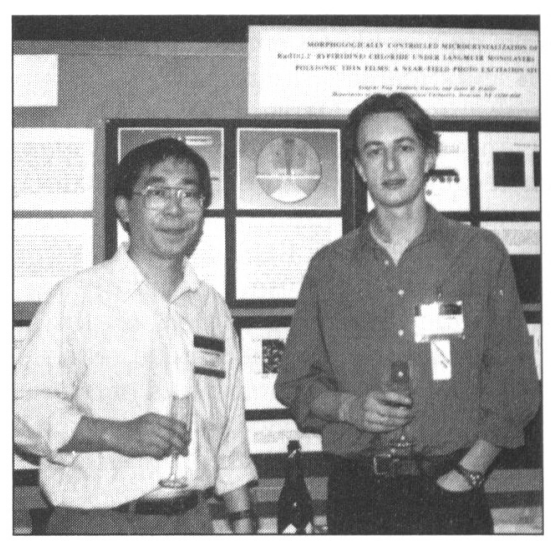

Tuesday (April 1) Poster Award recipient: Morphologically Controlled Microcrystalization and Electron Transfer of Ru(II)(2, 2'-Bipyridine) Chloride Under Langmuir Monolayers and In Polyionic Thin Films: A Near Field Photo-Excitation Study, Yongchi Tian, Frederic G. Guerin, Janos Hugo Fendler, Syracuse Univ, Dept of Chemistry, Syracuse, NY. (6.8)

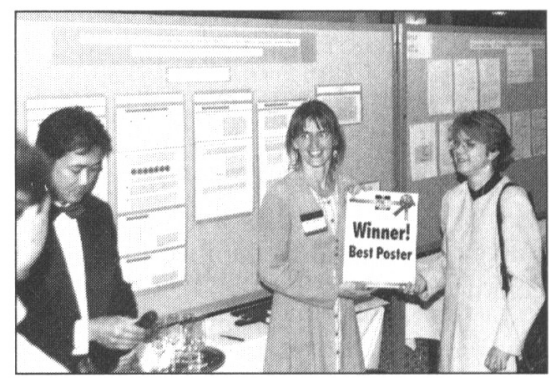

Thursday (April 3) Poster Award recipient: Magnetic and Magnetotransport Properties of p-Type (Ga,Mn)As, A New Diluted Magnetic Semiconductor, Ann Van Esch, Katholieke Univ. Leuven, Dept. of Physics, Leuven, Belgium; Jo De Boeck, IMEC, Dept. of MAP/MBE, Leuven, Belgium; Luc Van Bockstal, Ria Bogaerts, Fritz Herlach, Katholieke Univ. Leuven, Dept. of Physics, Leuven, Belgium; Gustaaf Borghs, IMEC, Dept. of MAP/MBE, Leuven, Belgium. (M10.8) (MRS chair Linda Griffith is seen to the right.) 
on microstructure defects in $\mathrm{SrTiO}_{3}$ bicrystals, and T. Kaplan (Conductus) discussing correlations between YBCO thin-film materials characteristics and $\mathrm{RF}$ device performance; (3) CMR oxide thin films and magnetic oxides, with N-C. Yeh (California Inst. of Technology) reporting on effects of controlled lattice distortion on the physical properties of magnetoresistive manganites and cobaltites; S. Jin (Bell Labs, Lucent Tech.) covering CMR in La-Ca-Mn-O; T. Venkatesan (Univ. of Maryland) discussing hybrid devices consisting of high-temperature cuprates and CMR manganites, $M$. Hundley (LANL) exploring the interplay between electronic transport and magnetic order in ferromagnetic manganite thin films; M. Jaime (Univ. of Dllinois_Urbana) discussing transport properties of CMR perovskites; S. Chambers (PNNL) reposing on oxygen-plasma-assisted MBE growth of iron oxides; and J. Kohlhepp (Univ. of Technology, Netherlands) presenting results on growth and STM of $\mathrm{Fe}_{3} \mathrm{O}_{4}$ on $\mathrm{MgO}$; (4) substrates for oxide epitaph, with C.D. Brandle (Bell Labs, Lucent Tech.) discussing substrate materials for high-temperature superconductors, and Q. Jiang (Univ. of Houston) presenting atomic scale engineering of $\mathrm{SrTiO}_{3}$ single crystal surfaces; and (5) oxide thin-film structure and growth, with J. Borchers (NIST) reporting on magnetic structure of transition-metal oxide superlattices; J-M. Triscone (Univ. de Geneve, Switzerland) discussing artificial doping in epitaxial heterostructures; and I. Bozovic (Varian Research Center) reporting on $\mathrm{MBE}$ growth of cuprate, manganite, and titanate heterostructures.

Symposium Support: NSF, ANL, LANL, Kurt J Lesker, Oxford Instruments, and HP.

\section{Thin Films Highlighted in the Field of Magnetic Materials \\ (See MRS Proceedings Volume 475)}

Symposium M on Magnetic Ultrathin Films, Multilayers, and Surfaces covered a broad range of topics in processing, structure, properties, and theoretical modeling. The Symposium began with a talk on magnetic measurements of microscopic quantities given by $\mathrm{S}$. Foner (MIT) and continued with a series of presentations on synthesis, processing and characterization of Heusler alloys, soft nitrides, and nanocrystals. In the session on novel applications and approaches for magnetism, the invited speaker, J. Heremans (GM R\&D) discussed that sensitivity/drift ratio is the important figure of merit for magnetic position sensors for automotive applications. Other papers on novel applications for magnetic systems included a paper presented by G. Strijkers (Eindhoven Univ. of Technology, Netherlands) on giant

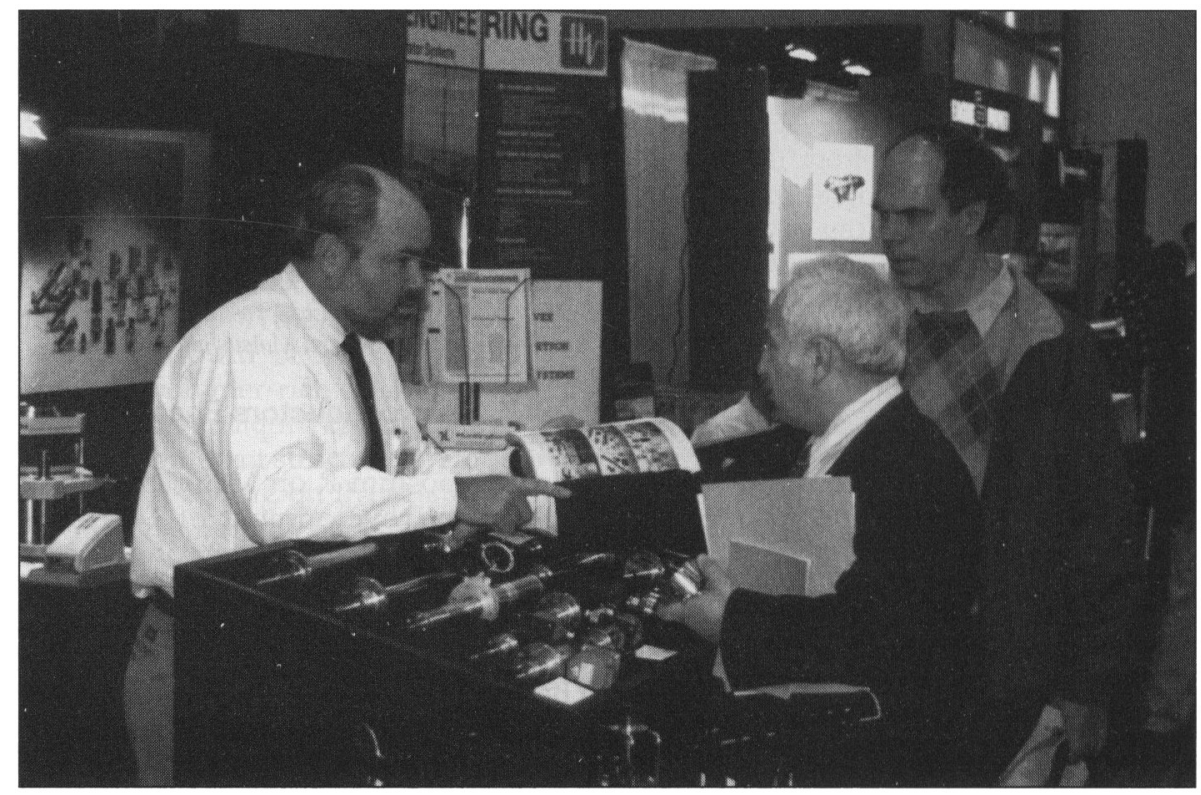

Over 120 exhibitors, including three entrepreneurs, displayed a full spectrum of equipment, instrumentation, products, software, publications, and senvices at the 1997 MRS Spring Meeting in San Francisco.

magnetoresistance induced by ultrathin Co layers. The session on nano/micro structure and magnetic properties emphasized the relationship of the magnetic properties of films to their structure determined by microscopy. Highlights included the invited talk by $R$. Wilson (IBMAlmaden) on the correlation of topography measured in the STM to properties of spin-valve structures; the demonstration by P. Galtier (Thomson CSF) that Fresnel contrast can permit the measurement of multilayer films even when there is very little Z-contrast, and the description by S. Hope (Cambridge Univ.) of an unusual adsorption-induced rotation of magnetic anisotropy. The invited speaker for the session on Structure and Properties: Mixing, Strain and Steps, P. Panissod (IPCMS, France), discussed the use of nuclear magnetic resonance technique in determining the local magnetic properties and structure of metallic multilayers. The session on magnetization reversal and domain structure, with invited talks by $B$. Barbara (CNRS, France) and C. Back (Stanford Univ.) demonstrated that significant progress has been made in the physical description of magnetic reversal in very small structures using new nanofabrication and physical observation methods such as MFM and magneto-optical Kerr effect. New results were also presented on the dynamics of domain wall motion. In the session on synchrotron radiation studies of magnetic materials, the techniques discussed ranged from $x$-ray absorption and photoelectron spectroscopy-often using polarization dichroism to obtain magnetic sensitivity - to nuclear resonant scattering. The invited speakers for the session were N. Smith (Advanced Light Source) and C. Schneider (Univ. of Mainz and the Max Planck Institute at Halle, Germany). Smith's talk on opportunities in research using synchrotron radiation was a natural introduction to a series of presentations on various forms of magnetically sensitive spectroscopies. Schneider's presentation on photoelectron microscopy was a highlight of the session, illustrating the potential of spectromicroscopy with the high resolution studies already performed by this group.

In the session devoted to magneto-optical properties and magnetic circular dichroism in thin films and multilayers, invited speakers by $\mathbf{H}$. Ebert (Univ. of Munich) reviewed the theory of magnetooptical effects and demonstrated that $a b$ initio calculations have lead to a basic understanding of these properties. The session clearly showed that the whole field has advanced extremely fast in the last few years. The session on SpinDependent Transport: CMR and Tunneling was in effect two sessions. Several talks, including an invited talk by G. Kwei (LANL), dealt with materials that exhibit CMR, with an emphasis on properties as a function of preparation conditions. An excellent and well-attended 
invited talk by S. Parkin (IBM-Almaden) launched a series of talks about spindependent tunneling. The invited talks in the final session of the symposium dealt with interlayer coupling from theoretical and experimental viewpoints, respectively. These works, presented by S. Mirbt (MIT) and J. Kohlhepp (Eindhoven Univ. of Technology, Netherlands) will undoubtedly trigger further research. A. Van Esch et al, (Catholic University of Leuven and IMEC, Belgium) received the MRS Meeting Chairs' poster award for their presentation on magnetic and magnetotransport properties of $p$-type $(\mathrm{Ga}, \mathrm{Mn}) \mathrm{As}$, a new diluted magnetic semiconductor (see sidebar on page 60 ).

Symposium Support: GMW Associates, MDC Vacuum Products, Ford Motor, US Inc., Lake Shore Cryotronics, Tencor Instruments, Komag, Physical Electronics, Princeton Measurements, MMR Technologies, Hitachi Central Research, Digital Instruments, Toshiba, and Fujitsu.

\section{The Silicon Industry Cleans Up Its Act (See MRS Proceedings Volume 477)}

Symposium P, Science and Technology of Semiconductor Surface Preparation, ran 12 sessions scanning the most important areas of surface cleaning and etching. T. Ohmi (Tohoku Univ., Japan) opened the symposium showing the chemical species formed during megasonic irradiation of water. In the next session, devoted to SC1 cleaning, W.C. Lee (Micron Tech.) showed a model for the adsorption and desorption of metals at the native oxide interface. S. Verhaverbeke (CFM Tech.) showed that the oxidation in $\mathrm{SC} 1$ is mainly due to the $\mathrm{HO}_{2}^{-}$ion. This was the first cleaning symposium where a special session was devoted to $\mathrm{CMP}$ cleaning. In the post-etch processing session, Y. Obeng (Bell Labs, Lucent Tech.) started by showing the different solvents and their problems now commonly used in the backend-of-the line. The surface microroughness session began with an excellent study by J. Rosamilia (Bell Labs, Lucent Tech.) on the roughening of silicon surfaces in DI-water. The gate oxide integrity session was dominated by papers showing that in a production environment, many different cleaning sequences result in similar oxide integrity. In the session on wet chemical cleaning and etching, A. Philipossian (Intel) showed an experimental model for the etching of $\mathrm{Ti}$ and TiN in SC1 solutions. Verhaverbeke followed by showing that the $\mathrm{HO}_{2}^{-}$anion is the main etchant, leading to some useful selectivity conclusions. The symposium was finished with a session on environmentally friendly processing. D. Riley (Advanced Micro Devices) showed how the recent knowledge on dilute SC-2 pro- cessing found their way into a production environment and T. Roche (Motorola) showed some ways to reduce rinsing water.

Symposium Support: Hitachi, NEC, SEMATECH, Ashland, Hoechst Celanese, Submicron Systems, Millipore, Morita Chemical Ind., Matsushita Electric Industrial Co., Santa Clara Plastics, DNS Electronics, SEMITOOL, Mitsubishi Electric Corp., Fujitsu, SONY, Tokyo Electron Ltd., Toshiba, Air Products and Chemicals, Fujikin, and Matsushita Electronics.

\section{High $\mathrm{T}_{\mathrm{c}}$ Superconductors Become More Flexible}

In Symposium $\mathrm{R}$ on Materials Issues Related to Development of Textured High-Temperature Superconducting Conductors, several papers dealt with possible approaches to the development of high current conductors utilizing $\mathrm{YBa}_{2} \mathrm{Cu}_{3} \mathrm{O}_{7}$ (Y123). The early success of $\mathrm{Bi}_{2} \mathrm{Sr}_{2} \mathrm{Ca}_{2} \mathrm{Cu}_{3} \mathrm{O}_{10}$ conductors manufactured by the powder-in-tube process and the continued improvement over the past several years in their properties has sustained interest in HTS conductor development, but the superior performance in high magnetic fields at high temperatures of single crystal Y123 films has appeared unattainable in long conductor geometry. Papers presented at this meeting provide new optimism that $Y 123$ conductors can be developed. Several approaches to preparation of highly textured, high-current density Y123 deposits on flexible metal substrates were discussed. All of the methods have in common epitaxial deposition of the superconductor on a highly textured oxide buffer layer on a metal substrate to obtain a highly textured superconducting film which has few weakly linked high-angle grain boundaries. The various approaches are distinguished by the method used to obtain the textured buffer layer. Approaches which have led to high-current density films include ion-beam-assisted deposition of the buffer, epitaxial deposition of the buffer on highly textured metal strip, and a method developed in Japan in which biaxially textured buffers are obtained by pulsed laser deposition by inclining the substrate relative to the ablation plume. Papers on the effects of angle of incidence on texture in other deposition processes also generated considerable interest.

Symposium Support: Southwire Co., EPRI, Univ. of Tennessee Space Institute, ORNL, and LANL.

\section{Optical Limiting Takes a Variety of Approaches}

(See MRS Proceedings Volume 479)

Materials for Optical Limiting (Symposium S) presented a broad technical scope covering organic and inorganic materials, liquid crystals, photorefractive materials, particle suspensions, and biological materials as well as characterization and device issues. In organic materials much interest centers on the phenomena of reverse saturable absorption and twophoton absorption. Phthalocyanines and porphyrins continue to receive a lot of attention, with a liquid form of phthalocyanine and modified forms of porphyrins showing interesting capabilities for optical limiting. Several two-photon materials exhibiting very large two-photon and excited state absorption cross sections were also introduced.

Processing of these organic materials in gels and sol-gel glasses offers new possibilities for devices. Liquid crystals continue to demand attention with new possibilities presented for optical limiting as well as tunable IR filters and switchable visible/near IR filters. IR optical limiting technology is approaching a stage of maturity with several exciting results reported for thermochromic and semiconductor materials. In general, the whole field is now making considerable advances with progress in new material design by computation, nonlinear optical propagation codes for designing devices, and demonstration devices validated in actual sensor protection tests.

Symposium Support: Air Force Wright Lab., Materials Directorate; and Defence Research Agency.

\section{Linking Computational Length and Time Scales}

The thrust of the papers in Symposium T, Computational Materials Science at the Mesoscale, was on the development of methods to describe materials behavior at the level of the microstructure. The presented work covered a wide range of materials topics, including simulations of individual dislocations, groups of dislocations, solidification, and grain growth. Techniques presented included molecular dynamics, Monte Carlo, phase fields, dislocation dynamics, and finite-element approaches. An important focus was on linking computational time and length scales.

The origin of recrystallization nuclei was discussed in a paper by A.D. Rollet (CMU) and W.W. Mullins (CMU) and E.A. Holm (SNL). A two-dimensional analysis showed that a single grain can grow abnormally when the boundary has certain combinations of energy and mobility. Monte Carlo Potts model simulations was used to show that the theoretical analysis is valid. Nucleation of recrystallized grains was discussed in terms of the coarsening of the subgrain network that then grows in an analogous way to abnormal grain growth.

A phase field model of dendritic solidi- 
fication in a binary alloy was presented by J.A. Warren (NIST). By incorporating a much smaller diffusion of the solute in the solid than the liquid, realistic macrosegregation patterns were obtained for the solid. The coalescence and fragmentation of dendritic side branches were examined.

One of the great challenges for materials modeling is the coupling of macroscopic response to microscopic properties. E. Tadmor et al. (Harvard Univ.) discussed their recent work connecting the finite-element method to atomistic simulations. He discussed recent advances in the methodology and presented a number of applications.

Symposium Support: SNL.

\section{Researchers Seek to Control Morphology}

The four-day symposium on Metastability and Critical Phenomenon in Polymer Phase Behavior (Symposium W) could be characterized as demonstrating the remarkable breadth of systems in which polymers, because of their relatively slow kinetics and relatively large length scales afford the possibility of fundamental detailed studies of phase transitions and design of technologically important structures. It is easy to strongly perturb polymer systems by the application of flow, electric, and magnetic fields and this leads not only to a richness of fundamental studies but to many practical technological applications such as polymer-dispersed LCDs and gels.

Polymer crystals typically have at least one dimension on the order of $10 \mathrm{~nm}$, thus anticipating via the Gibbs-Thomson equation a strong depression of the melting point. A. Keller (Univ. of Bristol) focused on the role of size-dependent metastability in crystalline polymers. $\mathrm{He}$ pointed out that the limited size of the newly forming crystal can cause an inversion of the stability of phases; that is, a phase which is metastable for an infinite size can become the stable phase when sufficiently small. Keller emphasized the profound influence such a situation can have on the development and control of chainfolded and chain-extended crystalline morphologies.

Another dominant theme throughout the symposium was a focus on how to control phase morphology through the interplay of the kinetics of ordering with the kinetics of phase separation in multicomponent blends. Particularly stimulating presentations were given by $\mathrm{A}$. Liu (UCLA) and by T. Bunning (Science Applications Intl. Corp.) which showed the wide range of structures possible in
PIPS. Liu's group employed generalized Cahn-Hilliard equations to follow the evolution of two order parameters: composition and orientation for a liquid crystalline-monomer system undergoing polymerization from an initially homogeneous nematic state. The morphology depends on the kinetics of polymerization versus the kinetics of phase separation as the growing polymers phase separate from the liquid crystal, forming an isotropic polymer-rich phase coexisting with a nematic liquid crystal-rich phase. Strikingly different structures were shown to arise depending on the relative rates. The systems become effectively arrested when the polymerization forms a threedimensional network.

Symposium Support: British Petroleum, Dutch State Mines, GE, Hoechst Celanese Corp., Philips Petroleum, 3M, and Wright Patterson AFB.

\section{Electron Microscopists Focus on Sample Preparation}

(See MRS Proceedings Volume 480)

By all measures, the focused ion beam technique received major attention at Symposium Z, the Workshop on Specimen Preparation for Transmission Electron Microscopy of Materials, with L. Giannuzzi (Univ. of Central Florida) speaking about its application to semiconductors and illustrating a novel liftout technique for thinned samples and $\mathrm{N}$. Rowlands (Micron Corp.) talking about its application to difficult-to-prepare galvanized steel samples. R. Alani (Gatan) spoke about low angle ion milling while J.S. Lee (LG Electronic Research Center, Korea) discussed the determination of the optimal rocking angle in an ion mill for multilayer systems. M. Cinibulk (Air Force Wright Lab.) showed the steps for preparing longitudinal and cross-sectional samples of fiber tows used in composites by the use of the Tripod Polisher which provides a large number of samples that are electron transparent. J. Grant (Univ. of Dayton) covered surface science aspects of TEM sample preparation. A highlight of his talk was the XPS analysis of intentionally contaminated samples cleaned by two commercially available plasma cleaners.

The question-and-answer period was sufficiently lively that it extended into and more or less started the scheduled Open Forum Discussion on the TEM sample preparation portion of the program. Although the Open Forum was intended to include any area of TEM sample preparation, two main topics of discussion evolved: sample contamination and FIB. The discussion on sample preparation centered on prevention and elimi-

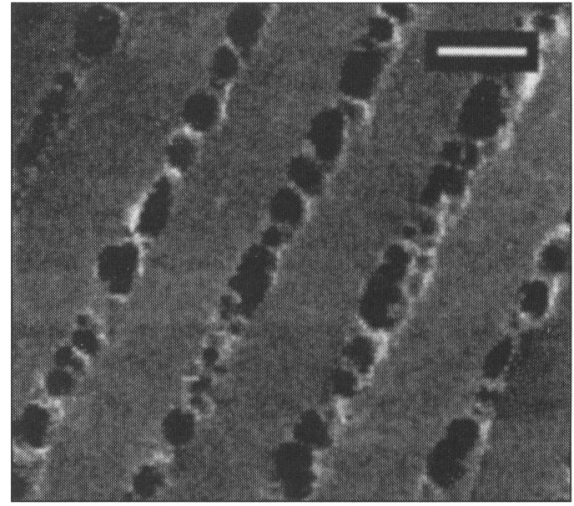

Photo polymerization within a standing wave pattem induces globally anisotropic PIPS structures. This is a $1.35 \mu \mathrm{m}$ Bragg spacing transmission grating formed through the anisotropic phase separation of liquid crystals in a polymer matrix. Scale bar corresponds to $1 \mu \mathrm{m}$. T. Bunning (Science Applications intl. Corp.) from Symposium W on Metastability and Critical Phenomenon in Polymer Phase Behavior explains that the simple idea is to use crossed laser beams to set up a spatially varying curing condition in an initially homogeneous nematic blend of monomer and liquid crystal. The relative balance of diffusion and reaction controls the morphology. Both reflective and transmission submicron size gratings could be produced. Surprisingly, microsecond response times were achieved for optical switching where the matched extraordinary index of the $L C$ regions is aligned with respect to the direction of the incident radiation. Preliminary work employing surfactants to tailor the anchoring conditions as well as the stability of the droplets within the liquid-crystal-rich layers is promising and shows opportunities for further development of this approach. See the summary on Symposium W.

nation and naturally led to a major discussion on plasma cleaners. For completeness and to round out the program, M. Boykin (Leica) was asked to present an application of ultramicrotomy to samples in the physical sciences. All manners of tips, tricks, and advice were exchanged by the audience participants from what grades of solvents to use to advice on how to apply the low-temperature wax commonly used in sample preparation.

Symposium Support: Wright Lab., Materials Directorate; Ted Pella Inc.; Micrion Corp.; Diatome U.S.; Delaware Diamond Knives; JEOL; Leo Electron Microscopy Inc;; Gatan; South Bay Technology Co.; Leica; FEI Co.; E.A. Fischione Instruments; IBM Analytical Services; NSA Hitachi; Philips Electronic Instruments; VCR Inc.; and Electron Microscopy Science. 